\title{
Graphdiyne: a two-dimensional thermoelectric material with high figure of
}

\section{merit}

L. Sun, P. H. Jiang, H. J. Liu*, D. D. Fan, J. H. Liang, J. Wei, L. Cheng, J. Zhang, J. Shi ${ }^{\dagger}$

Key Laboratory of Artificial Micro- and Nano-Structures of Ministry of Education and School of Physics and Technology, Wuhan University, Wuhan 430072, China

As a new carbon allotrope, the recently fabricated graphdiyne has attracted much attention due to its interesting two-dimensional character. Here we demonstrate by multiscale computations that, unlike graphene, graphdiyne has a natural band gap, and simultaneously possess high electrical conductivity, large Seebeck coefficient, and low thermal conductivity. At a carrier concentration of $2.74 \times 10^{11} \mathrm{~cm}^{-2}$ for holes and $1.62 \times 10^{11} \mathrm{~cm}^{-2}$ for electrons, the room temperature $Z T$ value of graphdiyne can be optimized to 3.0 and 4.8 , respectively, which makes it an ideal system to realize the concept of "phonon-glass and electron-crystal" in the thermoelectric community.

\footnotetext{
* Corresponding author. E-mail: phlhj@,whu.edu.cn (H. J. Liu)

Corresponding author. E-mail: jshi@,whu.edu.cn (J. Shi)
} 


\section{Introduction}

In the past several decades, plenty of efforts have been devoted to the fabrication and investigation of novel carbon allotropes, such as fullerene [1], carbon nanotube [2], and graphene [3]. In 2010, a new form of carbon allotrope named graphdiyne (GDY) was successfully synthesized on the surface of copper via cross coupling reaction [4]. GDY is a one-atom-thick two-dimensional material composed of $s p$ and $s p^{2}$ hybridized carbon atoms, where adjacent carbon hexagons are connected by two acetylenic linkages $(-C \equiv C-)$ [5]. Unlike graphene with a Dirac cone-like electronic structure, GDY has a natural band gap of $0.47 \mathrm{eV}[6]$. Due to its unique two-dimensional structure, GDY is predicted to be the most stable diacetylenic carbon allotropes with high thermal resistance, high electrical conductivity, extreme hardness, and it is synthetically approachable [7, 8]. Many potential applications of GDY have been proposed, including gas separation [9], lithium storage [10], catalyst for dehydrogenation [11], and replacement for the existing silicon transistor [12].

The high thermal resistance together with high electrical conductivity of GDY is reminiscent of thermoelectric materials, which can directly convert heat into electricity and vice versa. The efficiency of a thermoelectric material is determined by its figure of merit or the $Z T=S^{2} \sigma T /\left(\kappa_{e}+\kappa_{l}\right)$, where larger electrical conductivity $\sigma$ and Seebeck coefficient $S$ along with smaller thermal conductivity (including both electronic part $\kappa_{e}$ and lattice part $\kappa_{l}$ ) are required for better performance. An ideal thermoelectric material behaves as glass for phonons and crystal for electrons (PGEC), as first proposed by Slack [13]. To be competitive with the traditional energy 
conversion methods, the $Z T$ value of a thermoelectric material should be larger than 3.0. However, such target is still far from been reached. In this work, we combine first-principles calculations, Boltzmann theory, and molecular dynamics simulations to investigate the thermoelectric properties of GDY, and demonstrate that the room temperature $Z T$ value of this novel two-dimensional system can be optimized to 3.0 and 4.8 for the $p$-type and $n$-type carriers, respectively. Such high $Z T$ values significantly exceed most laboratory results reported so far, making GDY a plausible candidate for high-performance thermoelectric materials.

\section{Computational method}

The structure optimization and electronic properties of GDY are calculated by using the projector augmented wave (PAW) method within the framework of density functional theory (DFT). The code is implemented in the Vienna ab-initio Simulation Package $[14,15]$. We use the generalized gradient approximation (GGA) with the exchange-correlation energy in the form of Perdew-Burke-Ernzerhof (PBE) [16]. The cutoff energy of $400 \mathrm{eV}$ is chosen for the plane-wave expansion, and the Brillouin zones are sampled with a $11 \times 11 \times 1$ Monkhorst-Pack k-mesh. We adopt a standard supercell geometry where the GDY and its periodic images are aligned in a hexagonal array with large vacuum distance of $12 \AA$ so that they can be treated as independent entities. The atomic positions of the GDY are fully relaxed until the magnitude of the forces acting on all the atoms becomes less than $0.05 \mathrm{eV} / \AA$. The electronic transport coefficients $(S, \sigma)$ are evaluated by using the Boltzmann theory [17], where the 
relaxation time is estimated from the deformation potential (DP) theory [18]. For the phonon transport, we use the equilibrium molecular dynamics (EMD) simulations, where the carbon-carbon interactions are described by the adaptive intermolecular reactive empirical bond order (AIREBO) potential [19] and the time step is set to 0.1 fs. The system is first simulated in an NVT (constant number of atoms, volume, and temperature) ensemble for 1,000,000 steps, and then switched into an NVE (constant number of atoms, volume, and energy) ensemble for 500,000 steps to reach the equilibrium state. The heat current data are obtained from another 15,000,000 steps in the NVE ensemble and the thermal conductivity is calculated by using the Green-Kubo autocorrelation decay method [20, 21]. To ensure the accuracy of our results, the thermal conductivity is averaged over five simulations with different initial velocities. In addition, the size effect $[22,23]$ is considered and a $400 \AA \times 400$ A simulation cell containing 38,700 atoms is needed to obtain converged results.

\section{Results and discussion}

The crystal structure of GDY is shown in Figure 1, where the $s p$ and $s p^{2}$ hybridized carbon atoms are arranged in a hexagonal array. The optimized lattice constants are $a$ $=b=9.46 \AA$. The internal coordinates of three carbon atoms marked with $\mathrm{C}_{1}, \mathrm{C}_{2}$, and $\mathrm{C}_{3}$ are $(0.349,0.349,0.000),(0.201,0.201,0.000),(0.0708,0.0708,0.000)$, respectively. The coordinates of other fifteen carbon atoms within the unit cell can be derived by utilizing the hexagonal symmetry. The length of carbon-carbon bonds represented by $b_{1}, b_{2}, b_{3}, b_{4}$ are $1.432,1.396,1.233$ and $1.339 \AA$, respectively. Note 
that the length of $b_{1}$ and $b_{2}$ are similar to those found in graphene, while the values of $b_{3}$ are much smaller due to $s p$ hybridization of carbon atoms. These lattice parameters agree well with those found previously [6], and further confirms the reliability of our calculations. Figure 2 plots the energy band structure of GDY along the high symmetry lines in the hexagonal Brillouin zone. Unlike graphene with a zero gap, we see that GDY is a semiconductor with a direct band gap of $0.48 \mathrm{eV}$ (GGA-PBE value) at the $\Gamma$ point. Such natural gap is believed to be originated from the inhomogeneous $\pi$-bindings between differently hybridized carbon atoms, where the behavior in the chainsaw of GDY are different from those around the carbon hexagons [24, 25]. Moreover, we find that the valence band maximum (VBM) and the conduction band minimum $(\mathrm{CBM})$ are both doubly degenerate, which tends to increase the density-of-state effective mass, as will be discussed later. It should be noted that the standard DFT calculations usually underestimate the band gap when compared to the experimental value. One approach to overcome this deficiency is to calculate the quasiparticle properties with the GW approximation of the many-body effects [26]. Another possibility is using hybrids functionals such as Heyd-Scuseria-Ernzerhof functional (HSE) [27, 28], which also gives a better prediction for the band gap of many semiconductors [29]. In particular, it was previously found [30] that the band gap of graphdiyne is $1.10 \mathrm{eV}$ at the $\mathrm{GW}$ level, which is obviously larger than our calculated value at the GGA-PBE level. However, except for such difference in the band gap, we find that the band shape of these two kinds of calculations are almost identical to each other, especially for the energy bands around the Fermi level. As the 
actual value of band gap does not affect appreciably the thermoelectric properties [31, 32], and both the GW and HSE approaches are very time-consuming and huge computation resources are needed for transport coefficients calculations, we adopt the standard DFT calculations with GGA-PBE in the following discussions.

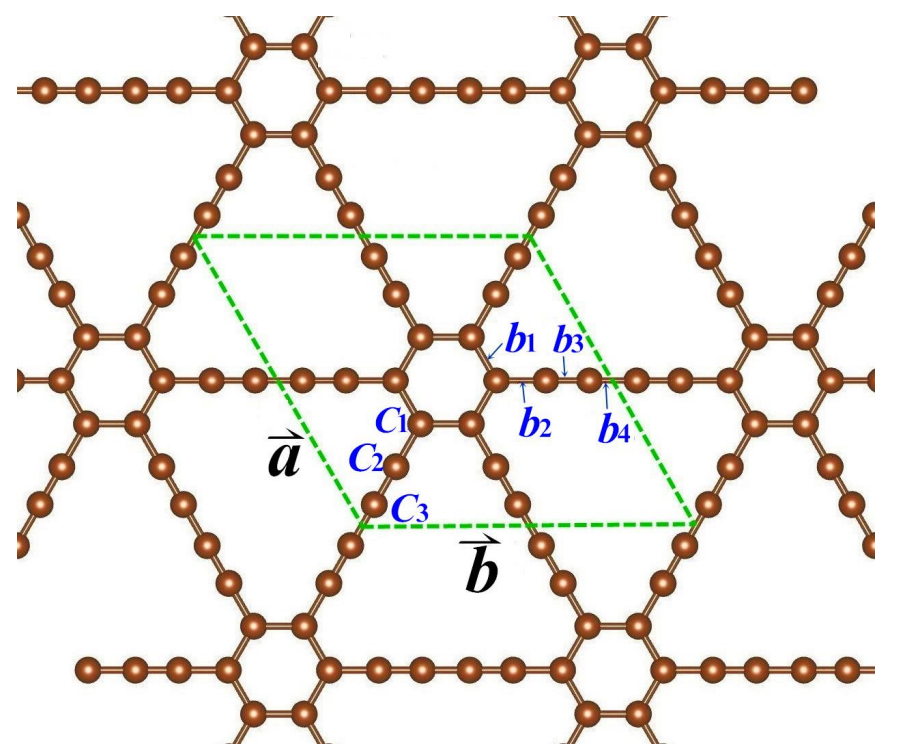

Figure 1. The ball-and-stick model of a single-layer GDY. The green dashed line indicates the unit cell with basis vector $\vec{a}$ and $\vec{b}$, where three typical carbon atoms are marked with $C_{1}, C_{2}$, and $C_{3}$, and $b_{1}, b_{2}, b_{3}, b_{4}$ represents different carbon-carbon bonds. 


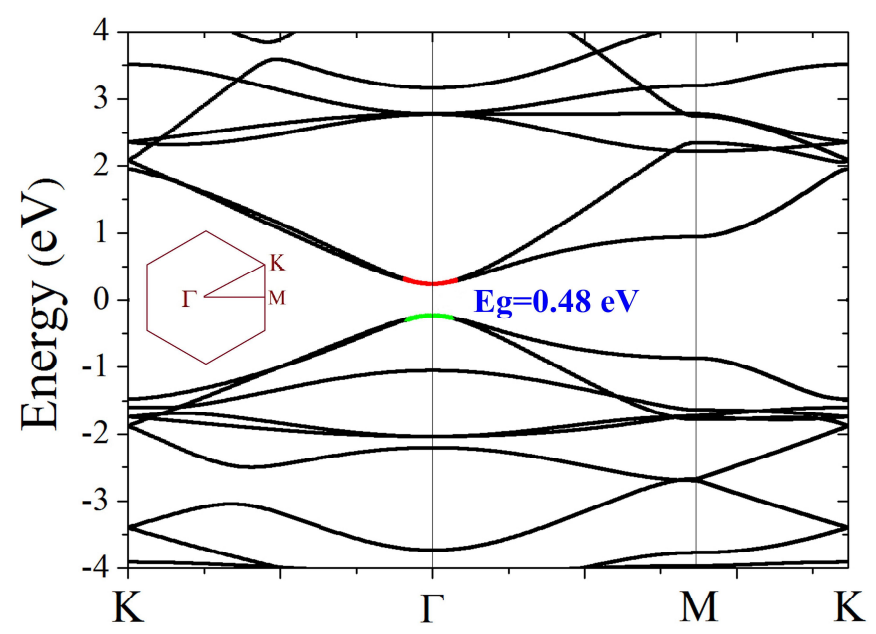

Figure 2. Band structure of GDY, where the CBM and VBM are both doubly-degenerate and are red- and green-colored, respectively. The inset shows the hexagonal Brillouin zone.

We now move to the investigations of transport properties. Using the semi-classical Boltzmann theory and relaxation time approximation [17], we can evaluate the electronic transport coefficients from the above-calculated energy band structures. Within this approach, the Seebeck coefficient $S$ is independent of relaxation time $\tau$, while the electrical conductivity $\sigma$ and the power factor $S^{2} \sigma$ are calculated with respect to $\tau$. Here the relaxation time is predicted from the DP theory [18] considering the acoustic phonons are the main scattering mechanism. For a two-dimensional system the relaxation time at temperature $T$ can be expressed as [33]:

$$
\tau=\frac{\mu_{c} m^{*}}{e}=\frac{2 \hbar^{3} C}{3 k_{B} T\left|m^{*}\right| E_{1}^{2}}
$$

where $\mu_{c}$ and $m^{*}$ are the carrier mobility and effective mass, respectively. Note here a density-of-state effective mass is used for $m^{*}$, which contains contributions from $k_{x}$ 
and $k_{y}$ directions, and the degeneracy of heavy and light bands at the $\Gamma$ point should be taken into account. The other parameters in Eq. (1) are the elastic constant $C$, the DP constant $E_{1}$, the unit charge $e$, the reduced Planck constant $\hbar$, and the Boltzmann constant $k_{B}$. For electrons and holes, we respectively consider the energy shift of CBM and VBM under uniaxial strain. Our calculated results are summarized in Table I. Compared with those of conventional thermoelectric materials, we see that the room temperature relaxation time of GDY is very large for both electrons and holes, which is very beneficial for the electronic transport and highly desirable for good thermoelectric performance. On the other hand, we see from Table I that the electrons exhibit even higher relaxation time than the holes. Since the density-of-state effective mass are similar for electrons and holes, such difference can be attributed to the smaller DP constant of electrons that arisen from the weak scattering by acoustic phonons [34].

Table I. The room temperature relaxation time $\tau$, carrier mobility $\mu_{c}$, DP constant $E_{1}$, elastic constant $C$, and density-of-state effective mass $m^{*}$ for GDY.

\begin{tabular}{cccccc}
\hline \hline \multirow{2}{*}{$\begin{array}{c}\mu_{c} \\
\text { Carrier type }\end{array}$} & $\tau$ & $E_{1}$ & $C$ & $m^{*}$ \\
electrons & 1.16 & 1.35 & 2.66 & 153.97 & 0.151 \\
holes & 0.46 & 0.51 & 4.15 & 153.97 & 0.159 \\
\hline \hline
\end{tabular}

Figure 3(a) shows the room temperature Seebeck coefficient $S$ of GDY at different carrier concentration $n$, where positive and negative $n$ indicate the electrons and holes, respectively. Note we are dealing with a two-dimensional system so that the 
concentration should be understood as the number of carriers per unit area. Around the Fermi level $(n=0)$, we see the Seebeck coefficient $S$ exhibit two obvious peaks, which is $-754 \mu \mathrm{V} / \mathrm{K}$ at $n=1.13 \times 10^{9} \mathrm{~cm}^{-2}$, and $756 \mu \mathrm{V} / \mathrm{K}$ at $n=-1.16 \times 10^{9} \mathrm{~cm}^{-2}$. The absolute values of these two Seebeck coefficients are much larger than those of most conventional thermoelectric materials, suggesting very favorable thermoelectric performance of GDY. However, we should note that the electrical conductivity $\sigma$ is actually very small at those small carrier concentrations, as shown in Figure 3(b). To maximize the power factor $\left(S^{2} \sigma\right)$, one therefore must try to find an optimized carrier concentration, which is shown in Figure 3(c). For $p$-type carriers, we see that the power factor can reach a maximum value of $0.21 \mathrm{Wm}^{-1} \mathrm{~K}^{-2}$ when the carrier concentration is tuned to $-1.5 \times 10^{12} \mathrm{~cm}^{-2}$. In the case of $n$-type carriers, an even higher power factor of $0.52 \mathrm{Wm}^{-1} \mathrm{~K}^{-2}$ can be achieved. Note that for low-dimensional systems such as the GDY, the definition of cross-sectional area or vacuum thickness has some arbitrariness. In the calculation of band structure of GDY, the supercell geometry has a vacuum thickness of $12 \AA$. However, to make better comparison with bulk systems (such as three-dimensional graphite), the electrical conductivity $\sigma$ and power factor $S^{2} \sigma$ of GDY are re-calculated (or converted) with respect to a "more realistic" vacuum thickness of $3.35 \AA$, which corresponds to the van der Waals distance of graphite and is generally adopted to evaluate the transport coefficients of two-dimensional carbon systems. 

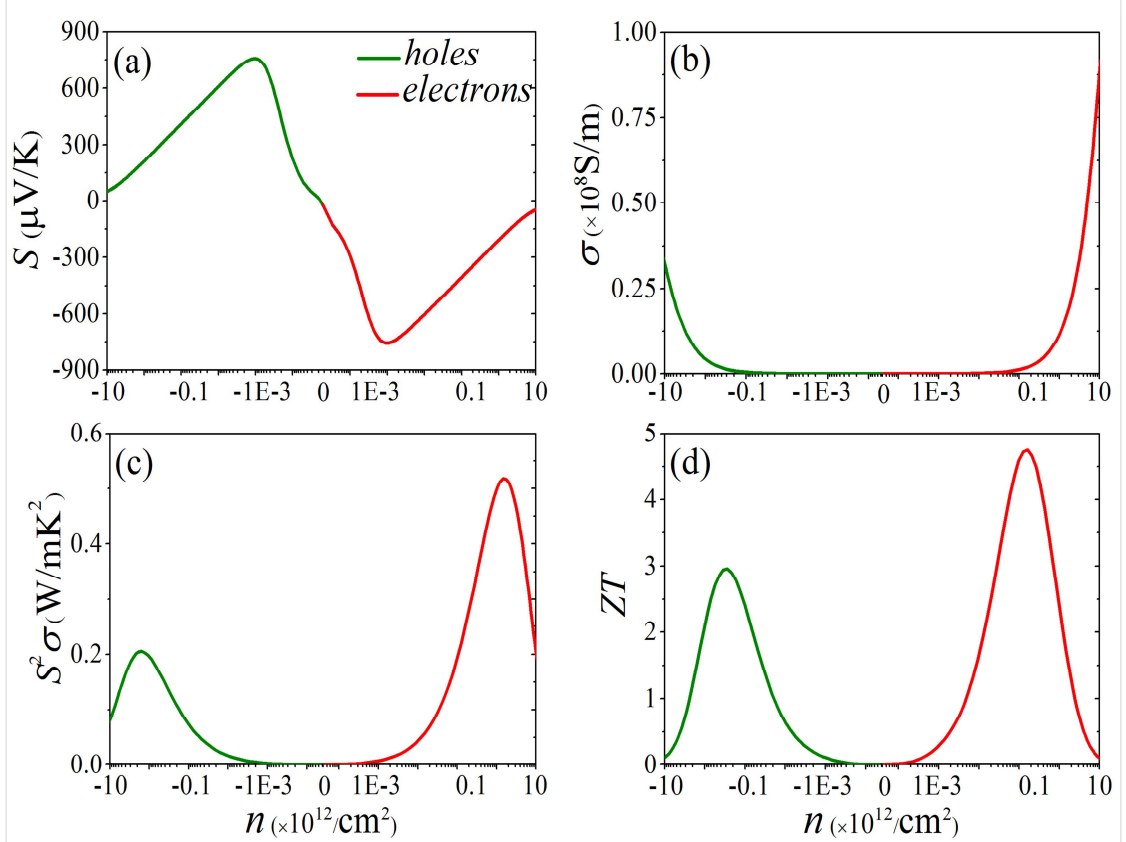

Figure 3. The room temperature Seebeck coefficient $S$, electrical conductivity $\sigma$, power factor $S^{2} \sigma$, and $Z T$ value of GDY as a function of carrier concentration $n$ (using logarithmic coordinates), where the green and red lines correspond to the holes and electrons, respectively.

We next discuss the heat transport coefficients of GDY, which consists of the electronic thermal conductivity $\kappa_{e}$ and the lattice part $\kappa_{l}$. In the present work, $\kappa_{e}$ is calculated by using the Wiedemann-Franz law [35]:

$$
\kappa_{e}=L \sigma T
$$

Here the Lorenz number $L$ for two-dimensional systems can be expressed as [36]:

$$
L=\left(\frac{k_{B}}{e}\right)^{2}\left[\frac{3 F_{2}}{F_{0}}-\left(\frac{2 F_{1}}{F_{0}}\right)^{2}\right]
$$

where $F_{i}$ is the Fermi integral:

$$
F_{i}=F_{i}(\eta)=\int_{0}^{\infty} \frac{x^{i} d x}{e^{x-\eta}+1}
$$


with $\eta=E_{f} / k_{B} T$ is the reduced Fermi energy. The calculated Lorenz number at optimized carrier concentration is listed in Table II.

Table II. The optimized room temperature $Z T$ values of GDY. The corresponding carrier concentration $n$, Seebeck coefficient $S$, electronic conductivity $\sigma$, Lorenz number $L$, electronic thermal conductivity $\kappa_{e}$, and lattice thermal conductivity $\kappa_{l}$ are also given. The parenthesized values are obtained using GW-corrected band gap, as compared with standard DFT calculations at GGA-PBE level.

\begin{tabular}{ccccccc}
\hline \hline$n$ & & $\sigma$ & $L$ & & & \\
$\left(\times 10^{11}\right.$ & $S$ & $\left(\times 10^{6}\right.$ & $\left(\times 10^{-8}\right.$ & $k_{e}$ & $k_{l}$ & $Z T$ \\
$\left.\mathrm{~cm}^{-2}\right)$ & $\left(\mu \mathrm{VK}^{-1}\right)$ & $\left.\mathrm{Sm}^{-1}\right)$ & $\left.\mathrm{V}^{2} \mathrm{~K}^{-2}\right)$ & $\left(\mathrm{Wm}^{-1} \mathrm{~K}^{-1}\right)$ & $\left(\mathrm{Wm}^{-1} \mathrm{~K}^{-1}\right)$ & \\
-2.74 & 323.90 & 1.20 & 1.52 & 5.46 & & 3.0 \\
$(-3.14)$ & $(323.94)$ & $(1.20)$ & $(1.52)$ & $(5.48)$ & & $(3.0)$ \\
1.62 & -363.46 & 1.91 & 1.51 & 8.62 & & 4.8 \\
$(1.59)$ & $(-379.21)$ & $(1.64)$ & $(1.50)$ & $(7.38)$ & & $(4.8)$ \\
\hline \hline
\end{tabular}

For the phonon transport of GDY, we use the EMD method where the lattice thermal conductivity $\kappa_{l}$ can be obtained by using the Green-Kubo relation [20, 21]:

$$
\kappa_{l}=\frac{1}{V k_{B} T^{2}} \int_{0}^{\tau_{m}}\langle J(\tau) J(0)\rangle d \tau
$$

Here $\langle J(\tau) J(0)\rangle$ is the heat current autocorrelation function, $V$ is the system volume, $k_{B}$ is the Boltzmann constant, and $T$ is the system temperature. As done for the electronic transport coefficients, we use the same vacuum thickness of $3.35 \AA$ to evaluate the lattice thermal conductivity of a two-dimensional system so that the $Z T$ value does not depend on the arbitrary definition of the cross-sectional area or vacuum thickness. In another word, if we use a different vacuum thickness (say, the 
initial value of $12 \AA)$, there will be changes of individual transport coefficients $\left(\sigma, \kappa_{e}\right.$, $\kappa_{l}$ ), while the $Z T$ value remains the same since the effect of arbitrariness is cancelled by the numerator and denominator of the $Z T$ formula. Our EMD simulation gives a lattice thermal conductivity of $7.30 \mathrm{Wm}^{-1} \mathrm{~K}^{-1}$ for GDY, which is much lower than those of other carbon allotropes such as graphene and carbon nanotubes, suggesting very favorable thermoelectric applications of GDY. The reduced thermal conductivity of GDY is believed to be caused by the presence of acetylenic linkages ( $s p$ carbon bond), which gives rise to inefficient heat transfer by lattice vibrations [37] compared with the $s p^{2}$ bonded carbon materials. Table II summarizes all the transport coefficients discussed above, from which we can now evaluate the thermoelectric performance of GDY. Figure 3(d) shows the room temperature $Z T$ values as a function of carrier concentration. At optimized concentration, we see that the $Z T$ value of GDY can be reached to 3.0 for $p$-type carriers and 4.8 for $n$-type carriers. These $Z T$ values significantly exceed most laboratory results reported so far, and are either equal to or larger than the target value $(Z T=3.0)$ for the commercial applications of thermoelectric materials. We want to emphasize that the above-calculated transport coefficients and $Z T$ values are based on the GGA-PBE band gap of $0.48 \mathrm{eV}$. If the more sophisticated GW technique is used, one can obtain an accurate band gap of $1.10 \mathrm{eV}$ [30]. To double-check that the actual value of band gap will not appreciably change the thermoelectric properties [31, 32], we make a rigid shift of the GGA-PBE band structure so that the band gap is increased to the $\mathrm{GW}$ value of $1.10 \mathrm{eV}$. At such accurate band gap, we find there are indeed very small changes of the optimized 
electronic transport coefficients (see the parenthesized values in Table II). The reason is that although increasing band gap may change specific value of transport coefficients (for example, increasing the peak value of Seebeck coefficient), the transport coefficients at the optimized concentration will not be changed substantially. In fact, we see from Table II that the GGA-PBE and GW approaches give identical ZT values for both the $n$-type and $p$-type GDY. In this regard, we believe the present calculations give a reasonable good prediction of the thermoelectric properties of GDY.

\section{Summary}

Using a multiscale approach combining first-principles, Boltzmann theory, and molecular dynamics simulations, we show that the recently fabricated GDY could be optimized to exhibit very high figure of merit. Our work not only provides a materials-specific system to realize the concept of PGEC, but also gives strong evidence that good thermoelectric performance can be achieved in previously unexpected carbon materials. It should be mentioned that single-layer GDY is currently only available as fractions in experiments [5], and great efforts should be devoted to the possible realization of this environmentally-friendly thermoelectric materials containing earth-abundant carbon element.

\section{Acknowledgements}

We thank financial support from the National Natural Science Foundation (Grant 
No. 51172167 and J1210061) and the "973 Program" of China (Grant No. 2013CB632502). 


\section{References}

[1] Kroto HW, Heath JR, O’Brien SC, Curl RF, Smally RE. C60: Buckminsterfullene. Nature 1985;318:162-3.

[2] Iijima S. Helical microtubules of graphitic carbon. Nature 1991;354(6348):56-8.

[3] Novoselov KS, Geim AK, Morozov SV, Jiang D, Zhang Y, Dubonos SV, et al. Electric filed effect in atomically thin carbon films. Science 2004;306(5696):666-9. [4] Li GX, Li YL, Liu HB, Guo YB, Li YJ, Zhu DB. Architecture of graphdiyne nanoscale films. Chem Commun 2010;46:3256-58.

[5] Haley MM, Brand SC, Pak JJ. Carbon networks based on dehydrobenzoannulenes: synthesis of graphdiyne substructures. Angew Chem Int Ed 1997;36:836-38.

[6] Pei Y. Mechanical properties of graphdiyne sheet. Physica B 2012;407:4436-39.

[7] Haley MM, Wan WB. Natural and non-natural planar carbon networks: from monomeric models to oligomeric substructures. Adv Strained Interesting Org Mol 2000;8:1-41.

[8] Balaban AT, Rentia CC, Gupitu E. Chemical graphs. VI. Estimation of relative stability of several planner and tridimensional lattices for elementary carbon. Rev Roum Chim 1968;13:231-47.

[9] Jiao Y, Du AJ, Hankel M, Zhu ZH, Rudolph V, Smith SC. Graphdiyne: a versatile nanomaterial for electronics and hydrogen purification. Chem Commun 2011;47:11843-5.

[10] Zhang HY, Xia YY, Bu HX, Wang XP, Zhang M, Luo YH, et al. Graphdiyne: a promising anode material for lithium ion batteries with high capacity and rate capability. J Appl Phys 2013;113:044309.

[11] Yu HZ, Du AJ, Song Y, Searles DJ. Graphyne and graphdiyne: versatile catalysts for dehydrogenation of light metal complex hydrides. J Phys Chem C 2013;117:21643-50.

[12] Peng Q, Dearden AK, Crean J, Han L, Liu S, Wen XD, et al. New materials graphyne, graphdiyne, graphone, and graphane: review of properties, synthesis, and application in nanotechnology. Nanotechnol Sci and Appl 2014;7:1-29.

[13] Slack GA. In: Rowe DM, editor. Handbook of Thermoelectrics, Boca Raton: CRC Press Inc;1995, p. 407. 
[14] Kresse G, Hafner J. Ab initio molecular dynamics for liquid metals. Phys Rev B 1993;47:558.

[15] Kresse G, Furthmüller J. Efficient iterative schemes for ab initio total-energy calculations using a plane-wave basis set. Phys Rev B 1996;54:11169.

[16] Perdew JP, Burke K, Ernzerhof M. Generalized gradient approximation made simple. Phys Rev Lett 1996;77:3865.

[17] Madsen GKH, Singh DJ. BoltzTraP. a code for calculating band-structure dependent quantities. Comput Phys Commun 2006;175:67-71.

[18] Bardeen J, Shockley W. Deformation potential and mobilities in non-polar crystals. Phys Rev 1950;80:72.

[19] Stuart SJ, Tutein AB, Harrison JA. A reactive potential for hydrocarbons with intermolecular interactions. J Chem Phys 2000;112:6472.

[20] Plimpton S. Fast parallel algorithms for short-range molecular dynamics. J Comput Phys 1995;117:1-19.

[21] Schelling PK, Phillpot SR, Keblinski P. Comparison of atomic-level simulation methods for computing thermal conductivity. Phys Rev B 2002;65:144306.

[22] Volz SG, Chen G. Molecular-dynamics simulation of thermal conductivity of silicon crystals. Phys Rev B 2000;61:2651-6.

[23] Che JW, Cagin T, Deng WQ, Goddard WA III. Thermal conductivity of diamond and related materials from molecular dynamics simulations. J Chem Phys 2000;113:6888-900.

[24] Bu H, Zhao M, Zhang H, Wang X, Xi Y, Wang Z. Isoelectronic doping of graphdiyne with boron and nitrogen: stable configurations and band gap modification. J Phys Chem A 2012;116(15):3934-9.

[25] He XJ, Tan J, Bu HX, Zhang HY, Zhao MW. The roles of p electrons in the electronic structures and optical properties of graphyne. Chin Sci Bull 2012;57(23):3080-3085.

[26] Onida G, Reining L, Rubio A. Electronic excitations: density-functional versus many-body Green's-function approaches. Rev Mod Phys 2002;74:601-59.

[27] Heyd J, Scuseria GE, Ernzerhof M. Hybrid functionals based on a screened Coulomb potential. J Chem Phys 2003;118:8207-15.

[28] Heyd J, Scuseria GE, Ernzerhof M. Erratum: "Hybrid functionals based on a 
screened Coulomb potential" [J. Chem. Phys. 118, 8207 (2003)]. J Chem Phys 2006;124:219906.

[29] Xiao H, Tahir-Kheli J, Goddard WA III. Accurate band gaps for semiconductors from density functional theory. J Phys Chem Lett 2011;2:212-7.

[30] Luo GF, Qian XM, Liu HB, Qin R, Zhou J, Li LZ, Gao ZX, Wang EG, Mei WN, Lu J, Li YL, Nagase S. Quasiparticle energies and excitonic effects of the two-dimensional carbon allotrope graphdiyne: theory and experiment. Phys Rev B 2011;84:075439.

[31] Fei RX, Faghaninia A, Soklaski R, Yan JA, Lo C, Yang L. Enhanced thermoelectric efficiency via orthogonal electrical and thermal conductances in phosphorene. Nano Lett 2014;14:6393-9.

[32] Wickramaratne D, Zahid F, Lake RK. Electronic and thermoelectric properties of few-layer transition metal dichalcogenides. J Chem Phys 2014;140:124710.

[33] Xi JY, Long MQ, Tang L, Wang D, Shuai ZG. First-principles prediction of charge mobility in carbon and organic nanomaterials. Nanoscale 2012;4:4348-69.

[34] Long MQ, Tang L, Wang D, Li YL, Shuai ZG. Electronic structure and carrier mobility in graphdiyne sheet and nanoribbons: theoretical predictions. ACS Nano 2011;5:2593-600.

[35] Bejan A, Allan AD. Heat Transfer Handbook. New York:Wiley, 2003:1338.

[36] Hikcs LD, Dresselhaus MS. Effect of quantum-well structures on the thermoelectric figure of merit. Phys Rev B 1993;47:12727.

[37] Zhang YY, Pei QX, Wang CM. A molecular dynamics investigation on thermal conductivity of graphynes. Comput Mater Sci 2012;65:406-10. 